\title{
Residual Stress Measurement with Laser-Optical and Mechanical Methods
}

\section{Venancio Martínez-García ${ }^{1, a^{*}}$, Martin Wenzelburger ${ }^{2, b}$, Andreas Killinger ${ }^{2, c}$, Giancarlo Pedrini ${ }^{3, \mathrm{~d}}$, Rainer Gadow ${ }^{2, \mathrm{e}}$ and Wolfgang Osten ${ }^{3, \mathrm{f}}$}

\author{
${ }^{1}$ Graduate School for advanced Manufacturing Engineering (GSaME), University of Stuttgart \\ ${ }^{2}$ Institut für Fertigungstechnologie keramischer Bauteile (IFKB), Institute for Manufacturing \\ Technologies of Ceramic Components and Composites, University of Stuttgart \\ ${ }^{3}$ Institut für Technische Optik (ITO), Institute of Applied Optics, University of Stuttgart \\ avenancio.martinez@gmail.uni-stuttgart.de, ㅎmartin.wenzelburger@ifkb.uni-stuttgart.de, \\ candreas.killinger@ifkb.uni-stuttgart.de, ${ }^{d}$ pedrini@ito.uni-stuttgart.de, \\ erainer.gadow@ifkb.uni-stuttgart.de, ${ }^{\mathrm{f}}$ osten@ito.uni-stuttgart.de
}

\begin{abstract}
Keywords: Hole-Drilling Method, Layer Composites, Residual Stress, Thermal Spraying, Laser, Digital Holographic Interferometry
\end{abstract}

\begin{abstract}
A new approach in hole-drilling residual stress analysis is described, applying a laser for quasi non-destructive material removal by laser ablation and measuring simultaneously the residual deformation around the hole by means of high-resolution, digital holographic interferometry. To evaluate this technology, an experimental bending device based on the European Standard for fourpoint bending tests was developed in order to prepare samples with a well-defined stress state by bending. This device was evaluated with the incremental-step hole-drilling/milling method. Residual stresses in thermal spray coatings were characterized by the hole-drilling/milling method, and residual strains were measured on the same coatings with the new, laser optical method. This paper describes preliminary results of work in progress.
\end{abstract}

\section{Introduction}

The hole-drilling and milling method, particularly in the form of mechanical machining of the drill hole by means of a circular drilling, milling or grinding operation, is a well-known technique for the measurement of residual stresses in material surfaces. However, measurements cause at least smallarea destruction of the sample component, and residual stress analysis is only possible in static condition after the component processing. Especially for coating application processes, the inprocess measurement of transient, process-induced stresses (thermal stresses, coating solidification stresses etc.) would lead to an increased understanding of residual stress evolution and could enable the manufacturing of coated parts with a residual stress state that lies within narrow limits of an application oriented, predefined residual stress regime. Requirements for this would be the possibility to perform large number, short interval in-process and non-destructive residual stress measurements.

This paper describes a new approach in hole-drilling residual stress analysis, applying a laser for material removal and measuring the residual deformation around the hole by optical methods, as well as the experimental setup for the method's evaluation by conventional, state of technology techniques. A laser is used for small area material removal by laser ablation, leading to residual deformation of the surface by stress relaxation and thermal influence of the absorbed laser energy. Surface deformations are measured simultaneously by means of high-resolution, digital holographic interferometry. Thermal and mechanical continuum simulation by the finite element method must be used to distinct the influences of residual stresses and of thermal deformation. In order to evaluate this technology on material surfaces of a well-known residual stress state, an experimental bending device was designed based on the European Standard for four-point bending tests, and experiments measuring well-defined in-plane stresses in curved strip specimen were carried out with the hole-drilling and milling technique as a reference. Results of these experiments are 
presented as well as a description of the measurement methods and a discussion of the difficulties, work in progress and potential of the method.

\section{Experimental residual stress analysis by the incremental-step, high-speed hole-drilling and circular milling method (reference technique)}

For the analysis of residual stresses in layer composites or in coatings, a variety of techniques is known. Many methods are laboratory measurement procedures, such as the diffraction methods, where X-ray, synchrotron or neutron radiation is applied, and therefore, large-scale analytical equipment is necessary. Results with a very high quantitative reliability can be achieved with the Xray diffraction method, where the measured crystal Bragg angle is influenced by crystal lattice deformations due to residual stresses, permitting the calculation of residual stresses of first, second and third (submicroscopic stresses on the atomic scale) order according to the theory of elasticity. For measurements on conductive or magnetic samples, methods based on eddy currents, the Barkhausen noise method or inductive measurements can be used. However, the required analysis times of these methods don't make them suitable for measurements during the coating process [1], [2].

In comparison, the hole-drilling technique (the conventional design or the more sophisticated technique with hole-drilling and circular milling) is a quasi-nondestructive method, which is used in practice with the advantages of time and cost efficient application and possibility of in-situ measurements even on large components, without losing depth resolution and quantitative accuracy. The residual stresses in the layer composite are locally relieved due to the material removal, which deforms the surface around the drill-hole. These deformations are measured through strain gages for every drilling step as relaxed, in-plane surface strains, which in combination with appropriate calibration curves (separately determined by simulation for layer composite, e.g. [3]) allow the quantitative determination of the residual stress state.

Through the combination of drilling or milling and small incremental vertical steps, residual stress/depth profiles can be determined. Disadvantages of this method are, e.g., that it can only be applied on flat and relatively smooth surfaces (due to practical reasons related with strain gage application), and that the method is restricted to the measurement of 2-dimensional deformations (lateral to the surface). Moreover, due to the size of the strain gage rosettes and the drilled hole, there are limitations both in terms of spatial resolution and in terms of practicability. Furthermore, these measurements are very error-prone to asymmetries of the hole or the positioning of the strain gage rosette [4].

\section{New approach in hole-drilling residual stress analysis}

Usually the strains released by hole-drilling are measured with strain-gage rosettes, but this involves the disadvantage of being a contact method since the sensor (strain gage) needs to be applied on the surface. Optical approaches based on speckle interferometry can be used as well for the determination of surface strains [5], [6]. A high-speed mechanical drilling/milling device may be used to produce the hole; however, in this paper we propose an approach with a laser used to drill the hole and an optical system based on holographic interferometry [7], [8] for the measurement of the resulting, 3-dimensional deformations.

The setup is shown in Fig. 1, where the beam emitted by a pulsed laser is focused on the coated object by using the lens L1. The wedge $\mathrm{W}$ produces a change of direction of the beam, and thus, its rotation allows to direct the focused beam at different locations of the sample and to form machine patterns that have, for example, the shape of a ring. The plate HS (harmonic separator) transmits the light coming from the pulsed laser having a wavelength of $1064 \mathrm{~nm}$ (near infrared). The object is illuminated by coherent green light (wavelength $532 \mathrm{~nm}$ ) which is scattered by the rough surface and reflected by the plate HS toward the holographic system where a lens images the surface to be investigated on a CCD camera with $2448 \times 2050$ pixels, pixel size $3.45 \times 3.45 \mu \mathrm{m}^{2}$. The sensor records the interference between the light scattered by the object and a reference beam from the same laser 
source used for illuminating the object. A deformation of the surface produces a phase change of the reflected beam, and digital holography allows to detect phase changes and retrieve the deformations with an accuracy of some tenths of a nanometer. Notice that for the measurement of 3-D deformations, the surface needs to be illuminated from at least three directions in order to obtain sensitivities along different vectors. In Fig. 1, only two illuminations (IL1 and IL2) are shown.

For measurements similar to the incremental hole-drilling method, the following procedure was applied:

- Recording of holograms before drilling by sequential object illumination from 4 directions.

- A pulsed laser with a pulse length of $10 \mathrm{~ns}$, repetition rate of 20 pulses/second and maximum pulse energy of $500 \mathrm{~mJ}$ is then used for machining (laser ablation) of the surface. The rotation of the wedge $\mathrm{W}$ displaces the beam focus and a ring-shape profile is produced. The depth of the profile depends on the laser pulse energy and the machining time.

- After milling, the holographic system measures the residual deformation (from these, the strain can be calculated).

- The pulsed laser is used again to increase the depth of the ring-shaped profile, and further holographic measurements are carried out.

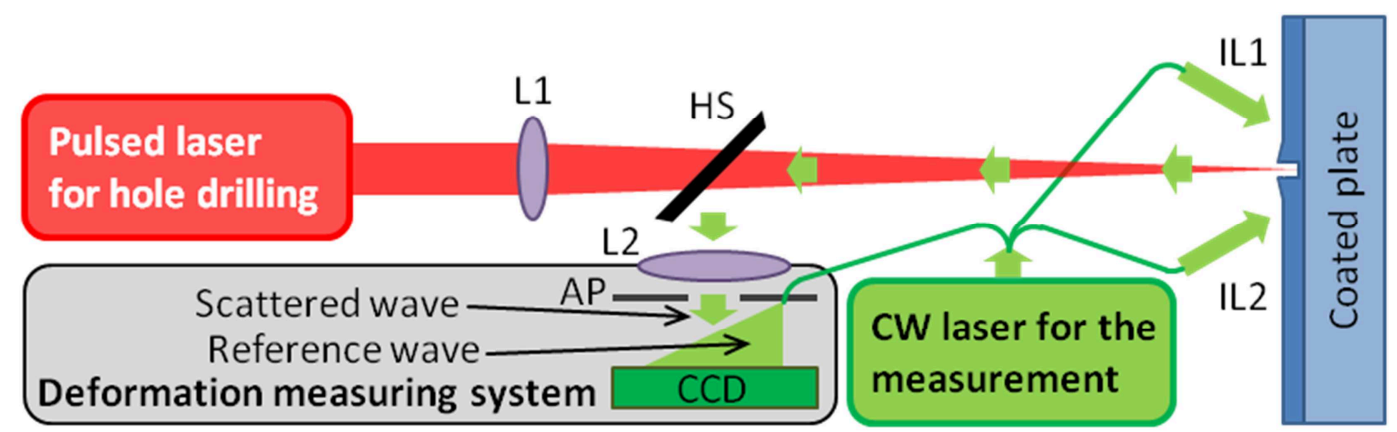

Fig. 1: Setup for hole-drilling by using a laser and measurement of the object's 3-dimensional surface deformation by digital holography.

\section{Results of measurements on thermal spray coatings}

Experimental investigations were performed on an alumina coated (aluminium oxide/titanium oxide) aluminium plate, first by the hole-drilling/milling method [9] as a reference, obtaining values for both principal residual stresses of around $600 \mathrm{MPa}$, as shown in Figs. 2 and 3. (Diameter of the hole was $1.8 \mathrm{~mm}$, grid center-line diameter of the strain gage rosette was $5.13 \mathrm{~mm}$.)

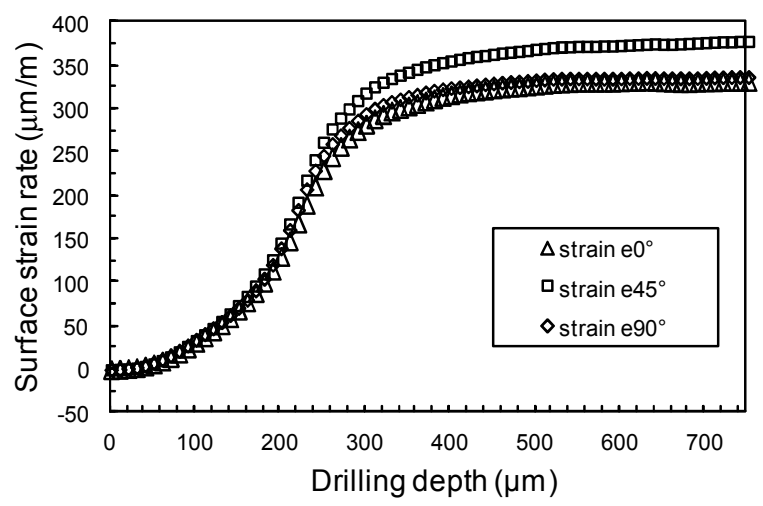

Fig. 2: Measured strain signals for the grid directions $\varepsilon_{1}\left(0^{\circ}\right), \varepsilon_{2}\left(45^{\circ}\right), \varepsilon_{3}\left(90^{\circ}\right)$.

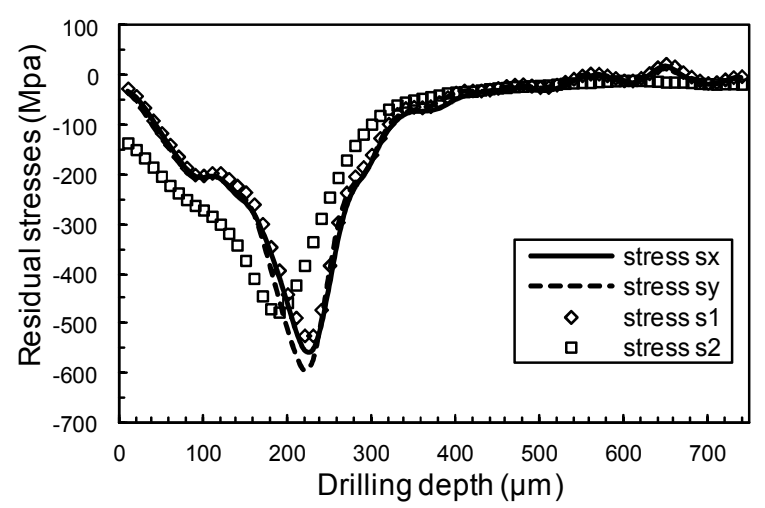

Fig 3: Calculated residual stresses; principal $\left(\sigma_{\mathrm{I}}, \sigma_{\mathrm{II}}\right)$ and specimen main directions $\left(\sigma_{\mathrm{x}}, \sigma_{\mathrm{y}}\right)$.

Measurement results (surface deformations due to relaxation of residual stresses) by the new method after laser ablation of a ring (radius approx. $600 \mu \mathrm{m}$, depth approx. $200 \mu \mathrm{m}$ ) are shown in Fig. 4. The laser ablation process produces in-plane and out-of-plane deformations around the ringshaped hole that are measured by holographic interferometry. The two images on the left ( $a$ and $b$ ) 
show phase maps of the in-plane deformations obtained by illumination of the surface from different directions. Coherent laser light was used for the illumination of the rough surface, and thus, the images are speckled. Moreover, laser ablation strongly changed the microstructure close to the ring, and this produces a de-correlation (the phase maps are very noisy around the ring). Therefore, results are given only for distances beginning at $1 \mathrm{~mm}$ from the center of the ring. The combination of the two phase maps results in the vectorial representation of in-plane deformations in Fig. 4 (c). There, the longest arrows (at $1 \mathrm{~mm}$ from the ring center) denote in-plane displacements of $150 \mathrm{~nm}$. The strain calculated from this value is $90 \mu \mathrm{m} / \mathrm{m}$. The highest measured out-of-plane deformation is $500 \mathrm{~nm}$ (also at $1 \mathrm{~mm}$ distance), see Fig. 4 (d).
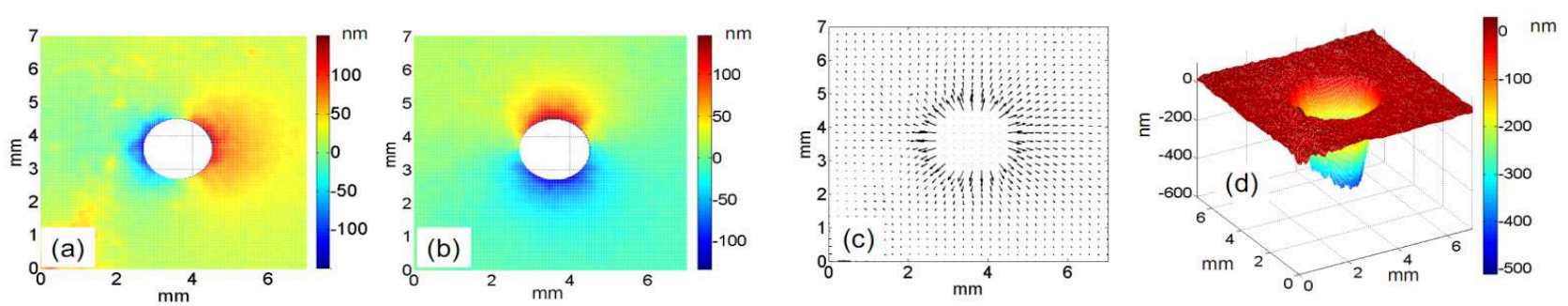

Fig. 4: Phase maps (in-plane deformations) obtained by illuminating the object from different directions (a: x-direction, and b: y-direction) and calculated vectorial in-plane deformations (c). Measured out-of-plane deformations (d).

Considering the different ratios of hole radius/measurement distance for the conventional and the introduced new method ( $r=R_{0} / R$, with values of 0.35 and 0.3 , resp.), the full-depth signal reduction for a dimensionless drilling depth of $Z / D=0.4$ (which is substantially higher than applied here) suggests higher measured strain values for the conventional method by a factor of approx. 1.3 [10]. At a depth of $200 \mu \mathrm{m}$, strain values with the conventional method are in the region of 100-125 $\mu \mathrm{m} / \mathrm{m}$, meaning that both methods yield very similar residual strain results. This shows the potential of the method for residual stress analysis after component processing. For in-process measurement of transient, process induced stresses by fast, simultaneous laser-ablation and deformation analysis, the speed of drill-hole application by laser ablation still needs to be increased.

\section{Bending device for method verification}

An experimental bending device was designed, based on [11], in order to provide samples with a well-known stress state for the verification of measurement accuracy. The reference describes a method for the determination of flexural strength of a test piece under three-point or four-point bending at room temperature, whereby four-point bending shows the advantage of a uniform stress level at the specimen's surface in-between the two inner supports. For the instrumentation described here, a well-defined bending load is applied manually and maintained at a constant value below the yield strength of the material. At mid-span, the bending moment is constant and the shearing force is zero, so that the maximum flexural stress $(\sigma f, \mathrm{~m})$ on the upper surface of the test specimen is

$$
\sigma_{f, m}=3 F_{m}\left(L-L_{i}\right) / 2 b h^{2}
$$

The inner supports have a span of $25 \mathrm{~mm}$, the outer supports of $75 \mathrm{~mm}\left(L_{\mathrm{i}}\right.$ and $L$, respectively). $F_{\mathrm{m}}$ is the maximum flexural force that is adjusted by moving the inner supports against the (fixed) outer supports, $b$ is the mean width, and $h$ is the mean thickness of the specimen. Load can be applied via the inner supports in both directions, thus enabling the measurement of tensile or compressive stresses on the specimen's upper surface. The maximum flexural force that can be applied is $1 \mathrm{kN}$, the maximum dimensions for the test specimen are $50 \mathrm{~mm}$ in width and $5 \mathrm{~mm}$ in thickness, with a minimum length of $100 \mathrm{~mm}$.

Prior to its application as a verification tool, the bending device was tested by measurements with the incremental-step, drilling and circular milling method, as described in [4], [9]. Specimen of aluminium $5754-\mathrm{H} 111(\mathrm{E}=68 \mathrm{GPa} ; \mu=0.34)$ were analysed, with dimensions of $100 \times 30 \times 5 \mathrm{~mm}^{3}$. 
To ensure that no residual stresses from prior material handling were present, a stress-relief heat treatment as specified by [12] was performed previously to stress application experiments.

Tensile stresses were measured with applied loads of $400 \mathrm{~N}$ and $800 \mathrm{~N}$, corresponding to surface stress values of $80 \mathrm{MPa}$ and $40 \mathrm{MPa}$, resp., according to Eq. 1. The measured strains are not shown here, but the respective, calculated residual stresses by the differential calculation method up to a drilling depth of $0.5 \mathrm{~mm}$ are shown in Fig. 8 (principal residual stresses $\sigma_{\mathrm{I}}$, equivalent residual stresses according to the maximum shear stress, or Tresca, theory, and the theoretical stress profile in bending). The principal residual stresses show some deviation from the theoretical stress/depth profile. For both tests, the deviation is bigger for the first steps, approaching the calculated curves with increasing drilling depth. However, the equivalent stress values are in much better agreement with the theoretical values. This is due to the fact that $\sigma_{\text {II }}$ was not zero, which indicates that the uniaxial bending tests resulted in a 2-dimensional stress distribution. Especially for $800 \mathrm{~N}$, which correlates with the material's minimum proof stress $\left(R_{\mathrm{p} 0.2}\right)$ of $80 \mathrm{MPa}$, the material may be subjected to non-proportional deformation.

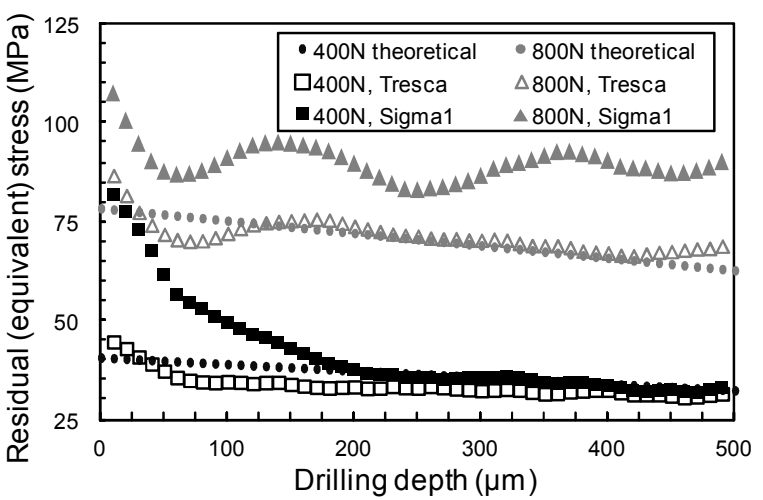

Fig. 8: Calculated residual stresses for $400 \mathrm{~N}$ and $800 \mathrm{~N}$ (principal and equivalent stresses).

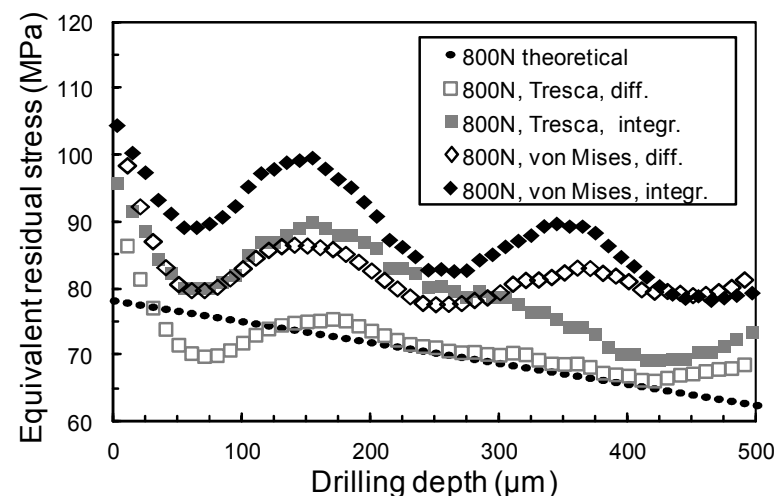

Fig 9: Comparison of stress calculation methods and equivalent stress theories.

To show the influence of the residual stress calculation method and equivalent stress theory used for residual stress analysis and visualization, the differential and integral methods as well as the maximum shear stress theory according to Tresca and the von Mises criterion (maximum distortion energy theory) are compared in Fig. 9 for the $800 \mathrm{~N}(80 \mathrm{MPa})$ test. Residual stress calculation with the differential method (after smoothing of the measured strain curves) and application of the maximum shear stress theory yields the best analogy with the theoretical stress curve.

\section{Summary and conclusions}

Residual stress analysis by the hole-drilling and milling method is a well-known technique, where the drill-hole is mechanically machined and the surface deformations are measured by a strain gage rosette. A new approach in hole-drilling residual stress analysis is described, applying a laser for quasi non-destructive material removal by laser ablation and measuring deformations around the hole by high-resolution, digital holographic interferometry. As an advantage, this method may enable in-process measurement of transient, process-induced stresses (thermal stresses, coating solidification stresses etc.) during coating application processes in the future, which would lead to an increased understanding of residual stress evolution and could enable the manufacturing of coated parts with a residual stress state that lies within narrow limits of an application oriented, predefined residual stress regime. First results showing the possibility of in-plane and out-of-plane deformation measurements with a high resolution are presented for a coated (mixed aluminium/titanium oxide coating material, atmospheric plasma spraying) aluminium test plate.

An experimental bending device was designed based on the European Standard for four-point bending tests, enabling the adjustment of well-defined in-plane stresses in curved strip specimens for the evaluation of residual stress measurement techniques. Measurements were carried out with the hole-drilling and milling technique, showing good agreement of the results for using equivalent 
stresses with the maximum shear stress theory, with deviations from the calculated stress level increasing at low drilling depths and close to the minimum proof stress. With stresses lower than the minimum proof stress (when a proportional deformation is ensured), measurements lead to very small errors, meaning that the device can be used for testing of residual stress measurement techniques.

\section{Acknowledgements}

This work was supported by the Deutsche Forschungsgemeinschaft DFG (German Research Foundation) under grant nos. GA589/10 and OS111/37.

\section{References}

[1] E. Macherauch, K.H. Kloos, "Origin, measurement and evaluation of residual stresses”, in: E. Macherauch, V. Hauk (Eds.): "Residual Stresses in Science and Technology" (Papers presented at the Int. Conf. on Residual Stresses, Garmisch-Partenkirchen, 1986), Vol. 1, DGM Informationsges., Oberursel, pp 3-26, 1987

[2] Robert B. Heimann, "Plasma-Spray Coating : Principles and Applications", Wiley-VCH, Weinheim, 2008

[3] B.-W. Hwang, C.-M. Suh, S.-H. Kim, "Finite element analysis of calibration factors for the modified incremental strain method", J. Strain Anal., 38, no. 1, pp 45-51, 2003

[4] M. Wenzelburger, D. López, R. Gadow, "Methods and application of residual stress analysis on thermally sprayed coatings and layer composites", Surf. Coat. Technol., 201 (5), pp 1995-2001, 2006

[5] M. Viotti, R. Suterio, A. Albertazzi, G. Kaufmann, "Residual stress measurement using a radial in-plane speckle interferometer and laser annealing: preliminary results", Opt. Lasers Eng., 42, pp 71-84, 2004

[6] M. Viotti, W. Kapp, A. Albertazzi, "Achromatic digital speckle pattern interferometer with constant radial in-plane sensitivity by using a diffractive optical element", Appl. Opt., 48, pp 22772281, 2009

[7] S. Seebacher, W. Osten, T. Baumbach, W. Jüptner, "The determination of material parameters of microcomponents using digital holography“, Opt. Lasers Eng., 36, 103-126, 2001

[8] G. Pedrini, W. Osten, and M.E. Gusev, "High-speed digital holographic interferometry for vibration measurement", Appl. Opt., 45, pp 3456-3462, 2006

[9] R. Gadow, M.J. Riegert-Escribano, M. Buchmann, "Residual Stress Analysis in Thermally Sprayed Layer Composites, Using the Hole Milling and Drilling Method", J. Therm. Spray Technol., 14 (1), pp 100-108, 2005

[10] Vishay Measurements Group, Vishay Micro-Measurements, "Tech Note TN-503 : Measurement of Residual Stresses by the Hole-Drilling Strain Gage Method" (Document Number: 11053; Revision: 15. Aug. 2007)

[11] European Standard "EN 658-3:2002-11, Advanced technical ceramics - Mechanical properties of ceramic composites at room temperature - Part 3: Determination of flexural strength"

[12] German Standard "DIN 65582:1990-12, Aerospace; heat treatment of aluminium- and magnesium-alloy castings" 